Supporting Information for the manuscript

\title{
Differential Line Broadening in MAS solid-state NMR due to Dynamic Interference
}

by

\begin{abstract}
Veniamin Chevelkov, Katja Faelber, Anna Schrey, Kristina Rehbein, Anne Diehl and
\end{abstract} Bernd Reif

\section{Interference of ${ }^{15} \mathrm{~N}-\mathrm{CSA}$ and ${ }^{1} \mathrm{H},{ }^{15} \mathrm{~N}$ dipole in the static case}

In the static case, the dynamic frequency $\omega_{\mathrm{N}}{ }^{\mathrm{PAS}}$ in the nitrogen principal axis system is determined by two contributions due to the ${ }^{1} \mathrm{H},{ }^{15} \mathrm{~N}$ dipolar interaction and the ${ }^{15} \mathrm{~N}$ chemical shielding. $\omega_{\mathrm{N}}^{\text {PAS }}$ can be written as

$$
\begin{aligned}
\omega_{N}^{P A S}(\beta) & =\omega_{N}^{C S A}(\beta)+\omega_{N H}^{\text {Dipol }}(\beta) \\
& =\left[\sigma_{N}^{i s o}+\delta_{N} \frac{3 \cos ^{2} \beta-1}{2}\right] N_{z}+D_{H N} \frac{3 \cos ^{2} \beta-1}{2} 2 N_{z} H_{z} \quad \text { [Eq. 1] } \\
& =\sigma^{i s o} N_{z}+\frac{3 \cos ^{2} \beta-1}{2}\left(\delta_{N}+2 D_{H N} H_{z}\right) N_{z}
\end{aligned}
$$

with

$$
D_{H N}=\left(\frac{\mu_{0}}{4 \pi}\right) \frac{\gamma_{H} \gamma_{N} \hbar}{r_{N H}^{3}}
$$

in which $\sigma^{\text {iso }}$ and $\delta$ describe the isotropic and anisotropic chemical shift of the nitrogen spin, respectively. $\beta$ refers to the angle of the principal axis of the dipolar / shielding tensor with respect to the external magnetic field. For simplicity, it is assumed that the dipolar and chemical shielding tensors are collinear and that the ${ }^{15} \mathrm{~N}$ shielding tensor is axially symmetric. $D_{\mathrm{HN}}$ represents the size of the ${ }^{1} \mathrm{H},{ }^{15} \mathrm{~N}$ dipolar interaction which is dependent on the magnetic permeability $\mu_{0}$, the gyromagnetic ratio $\gamma_{\mathrm{H}}$ and $\gamma_{\mathrm{N}}$ of the proton and the nitrogen nucleus, respectively, Planck's constant $\hbar$, and the $\mathrm{N}-\mathrm{H}$ bond length $r_{\mathrm{NH}}$. Given the fact, that the proton spin state can adopt the spin quantum number $\pm 1 / 2$, one obtains 


$$
\begin{aligned}
\delta_{N}+D_{H N} H_{z} & =\delta_{N}+D_{H N}\left[\left(\frac{1}{2}+H_{z}\right)-\left(\frac{1}{2}-H_{z}\right)\right] \\
& =\delta_{N}+D_{H N}\left[H^{\alpha}\right]-D_{H N}\left[H^{\beta}\right] \\
& =\left\{\begin{aligned}
\delta_{N}+D_{H N}\left[H^{\alpha}\right] & ; \text { upfield component } \\
\delta_{N}-D_{H N}\left[H^{\beta}\right] & ; \text { downfield component }
\end{aligned}\right.
\end{aligned}
$$

As a consequence, the $\beta$ spin-state of the multiplett experiences effectively only the difference anisotropy $\delta_{\mathrm{N}}-D_{\mathrm{HN}}$, whereas the $\alpha$ spin-state experiences the sum anisotropy $\delta_{\mathrm{N}}+D_{\mathrm{HN}}$. The intensity of the resonance associated with the sum is therefore distributed over a larger spectral region and the respective central band intensity is decreased. The opposite applies for the resonance associated with the difference tensor.

\section{Definition of CSA Tensor Properties}

We use the convention of Haeberlen ${ }^{1,2}$ in order to describe ${ }^{15} \mathrm{~N}$ chemical shift tensor properties. The reduced anisotropy $\delta_{\mathrm{z}}$ denotes the difference between the most downfield component in the static powder pattern $\delta_{\mathrm{zz}}$ and the isotropic chemical shift $\delta_{\text {iso }}, \delta_{\mathrm{z}}=\delta_{\mathrm{zz}}-\delta_{\text {iso }}$, with $\delta_{\text {iso }}=1 / 3\left(\delta_{\mathrm{xx}}+\delta_{\mathrm{yy}}+\delta_{\mathrm{zz}}\right) \cdot \eta$ refers to the asymmetry of the tensor, $\eta=\left(\delta_{\mathrm{yy}}-\delta_{\mathrm{xx}}\right) /\left(\delta_{\mathrm{zz}}-\right.$ $\left.\delta_{\text {iso }}\right)=\left(\delta_{\mathrm{y}}-\delta_{\mathrm{x}}\right) / \delta_{\mathrm{z}}$, asuming $\left|\delta_{\mathrm{zz}}-\delta_{\text {iso }}\right| \geq\left|\delta_{\mathrm{xx}}-\delta_{\text {iso }}\right| \geq\left|\delta_{\mathrm{yy}}-\delta_{\text {iso }}\right| . \beta$ represents the angle between the principal axis of the ${ }^{15} \mathrm{~N},{ }^{1} \mathrm{H}$ dipolar and the ${ }^{15} \mathrm{~N}$ chemical shift tensors. The chemical shift anisotropy $\Delta \delta$ which is commonly used for the description of solution-state NMR experiments is defined as $\Delta \delta=3 / 2 \delta_{\mathrm{z}}=\delta_{\mathrm{zz}}-1 / 2\left(\delta_{\mathrm{xx}}+\delta_{\mathrm{yy}}\right)$. 


\section{Investigation of the influence of Magic Angle missettings on solid-state NMR spectra}

A major concern in the experiments is the proper adjustment of the magic angle. Missetting of this angle reintroduces anisotropic interactions and yields a dramatic deterioration of the line shape of the broad component of the spectrum. This effect is illustrated in supporting figure 1.

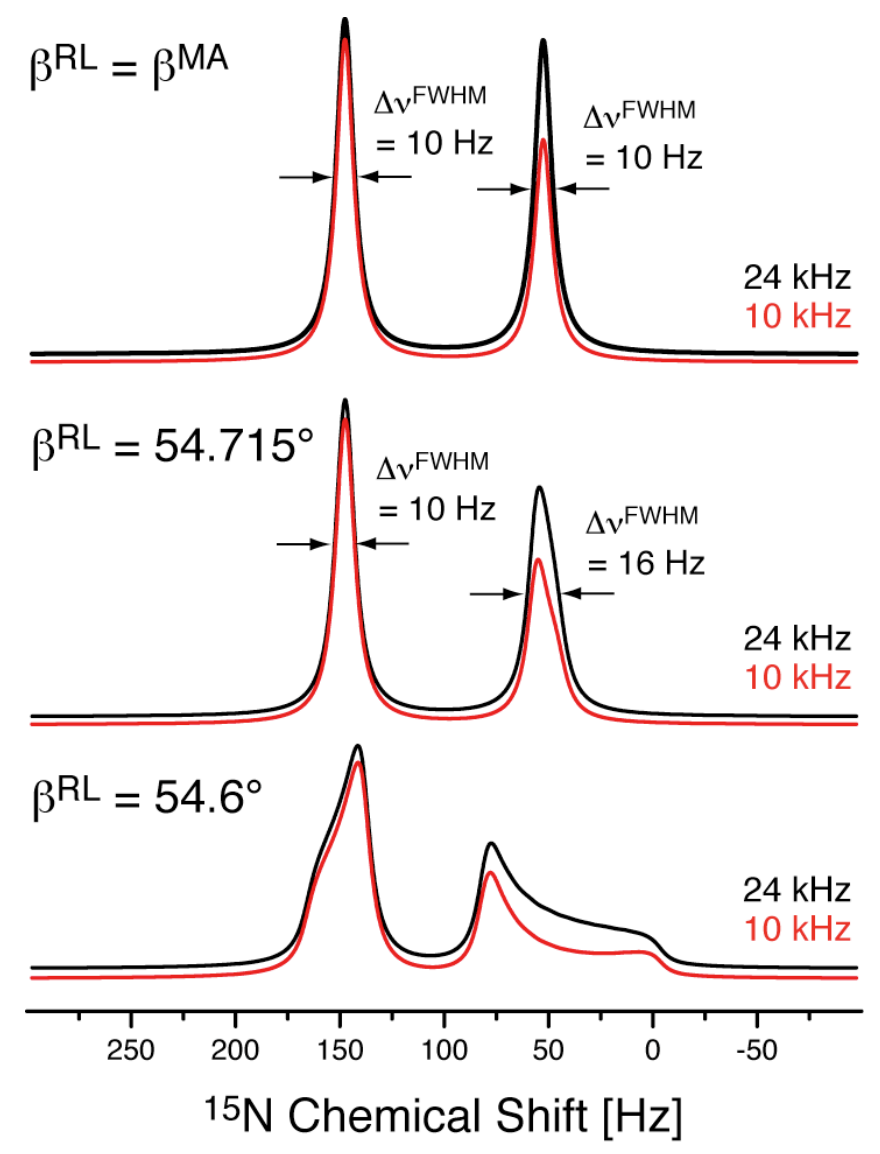

Supporting Figure 1. Theoretical ${ }^{15} \mathrm{~N}$ spectrum assuming a chemical shift anisotropy of $100 \mathrm{ppm}$ and a dipolar and scalar coupling to a directly bonded proton of $10 \mathrm{kHz}$ and $-95 \mathrm{~Hz}$, respectively, and an external magnetic field of $600 \mathrm{MHz}$. Simulations are shown for a MAS rotation frequency of $10 \mathrm{kHz}$ (red) and $24 \mathrm{kHz}$ (black). The simulation is carried out for three different values for the Euler angle $\beta^{\mathrm{RL}}$ which describes the angle between the principal axes of the rotor fixed frame and the laboratory coordinate system. Missetting of the "magic angle" $\left(\beta^{M A}=\beta^{R L}=\arctan (\sqrt{2}) \approx 54.73561\right)$ reintroduces the sum and difference anisotropy for the upfield and downfield component in the spectrum, respectively.

The magic angle missettings in the simulations represented in supporting figure 1 are largely exagerated compared to the achieved accuracy in the experimental setup. A magic angle missetting of $0.0206^{\circ}$ results in a differential line width of $6 \mathrm{~Hz}$. The minimum differential line width observed in our experiments amounts to $1 \mathrm{~Hz}$ (at a MAS frequency of $24 \mathrm{kHz}$ and an effective temperature $T^{\mathrm{eff}}=24^{\circ} \mathrm{C}$ ). 


\section{Effects of variations of the size and orientation of the ${ }^{15} \mathrm{~N}$ CSA tensor on the ${ }^{15} \mathrm{~N}-\mathrm{H}^{\alpha} /{ }^{15} \mathrm{~N}-$ $\mathbf{H}^{\beta}$ differential multiplet intensities}

The ${ }^{15} \mathrm{~N}$ CSA tensor in peptides and proteins was quantified previously. ${ }^{2-5}$ Typical values for $\delta_{\mathrm{z}}$ and $\eta$ were found to be on the order of $\delta_{\mathrm{z}}=(100 \pm 5) \mathrm{ppm}, \eta=0.4 \pm 0.05$ was found. The angle among the $\mathrm{N}-\mathrm{H}$ bond vector and the principal axis of the ${ }^{15} \mathrm{~N}$ CSA tensor is determined to be around $21^{\circ} .5$ Our simulations show that under these conditions, the $\mathrm{NH}^{\alpha / \beta}$ intensity ratio yields a value of 1.31-1.37 which is within experimental error. The asymmetry parameter $\eta$ does not influence the cross term effect.

A)

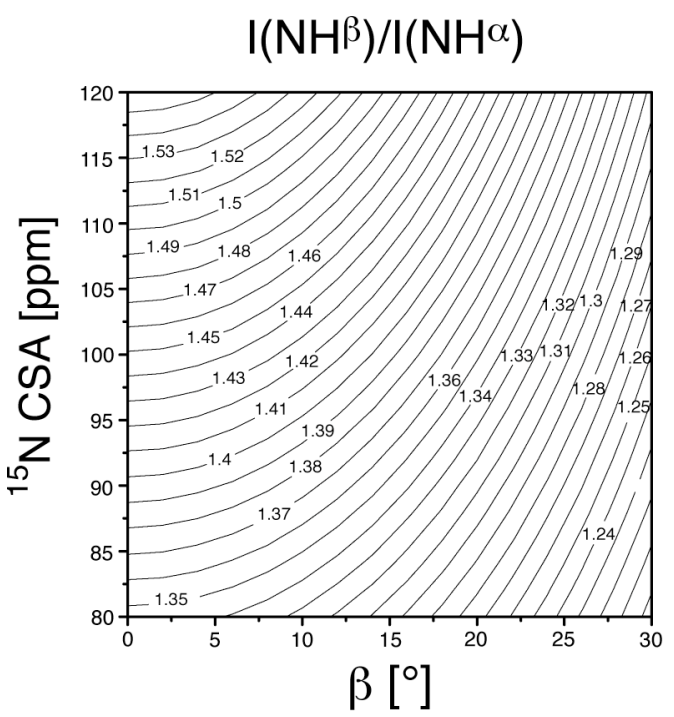

B)

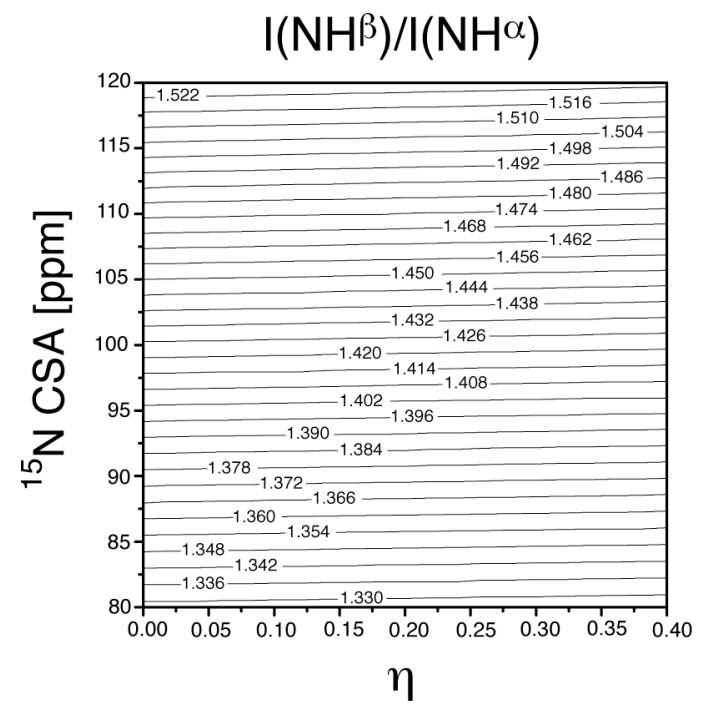

Supporting Figure 2. A) Simulation of the $\mathrm{NH}^{\alpha} / \mathrm{NH}^{\beta}$ intensity ratio of the nitrogen signal as function of the anisotropy $\delta_{z}$ and the angle $\beta$ at a MAS frequency of $10 \mathrm{kHz}$ (the asymmetry is kept constant at $\eta=0.2$ ). B) Simulation of the $\mathrm{NH}^{\alpha} / \mathrm{NH}^{\beta}$ intensity ratio of the nitrogen signal as a function of the reduced anisotropy $\delta_{\mathrm{z}}$ and the asymmetry $\eta$ at a MAS frequency of $10 \mathrm{kHz}$ (the $\beta$ angle is kept constant at $\beta=10^{\circ}$ ). In both simulation, an external magnetic field of $14.1 \mathrm{~T}$ is assumed, corresponding to a ${ }^{1} \mathrm{H}$ Larmor frequency of $600 \mathrm{MHz}$.

An analysis of the MAS frequency dependence of the $\mathrm{HN}^{\alpha / \beta}$ intensity ratio from Fig. 5 in the main manuscript allows in principle to extract the angle $\beta$ between the principal axis of the ${ }^{1} \mathrm{H},{ }^{15} \mathrm{~N}$ dipolar and ${ }^{15} \mathrm{~N}$ CSA interaction tensors. In order to extract this parameter accurately, higher precision measurements have to be carried out, however. 


\section{Sample heating due to friction induced by sample spinning}

The effect of MAS and application of RF pulses on the sample temperature under various salt concentrations was investigated recently in a number of studies. ${ }^{6-10} \mathrm{We}$ determined the heating effect due to MAS independently, making use of the strong temperature dependence of the water resonance. The temperature dependent chemical shift of $\mathrm{H}_{2} \mathrm{O}$ is empirically given as (supporting figure $3 \mathrm{~A}$ )

$$
\delta_{H 2 O}[\mathrm{ppm}]=8.33-0.01179 \cdot T[\mathrm{~K}]
$$

This calibration is similar to the one obtained previously. ${ }^{11,12}$ Using this calibration, we determined the MAS induced temperature increase in the NMR rotor. A change in the MAS rotation frequency from 13 to $24 \mathrm{kHz}$ induces an increase in temperature of approximately 20 ${ }^{\circ} \mathrm{C}$ (Supporting figure 3B).

A)

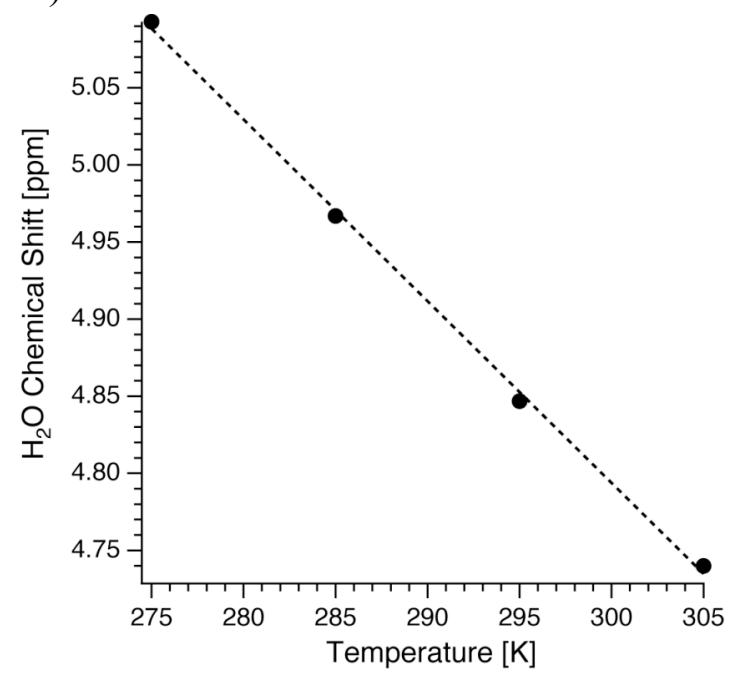

B)

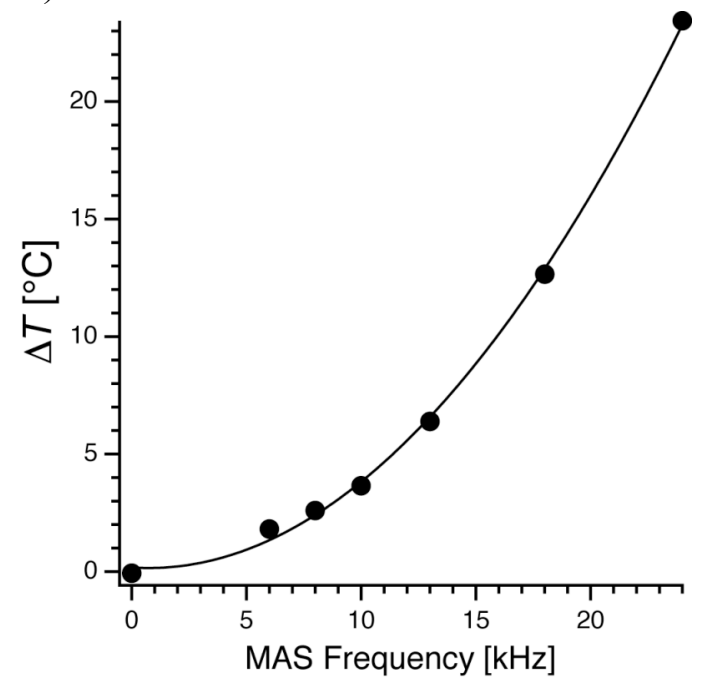

Supporting Figure 3. A) Calibration of the $\mathrm{H}_{2} \mathrm{O}$ chemical shift as a function of the external temperature. B) Increase of sample temperature as a function of MAS rotation frequency.

\section{NMR Pulse Sequences}

In order to record ${ }^{1} \mathrm{H},{ }^{15} \mathrm{~N}$ correlation experiments, either cross polarization $(\mathrm{CP})$ or INEPT transfers can be employed. In our hands, both transfer schemes yield approximately the same signal to noise ratio. The RF pulse sequence used to select for one of the proton spin states in the nitrogen evolution period (IPAP) ${ }^{13,14}$ is depicted in supporting figure 4. Variation of the delays $\varepsilon_{1}$ and $\varepsilon_{2}$ together with the phases $\phi_{2}$ and $\phi_{\text {rec }}$ results in acquisition of an in-phase or 
antiphase multiplet pattern (see figure caption for description). Addition or subtraction of the two sub-spectra yields exclusively the downfield or upfield component of the ${ }^{15} \mathrm{~N}$ doublet.

A)

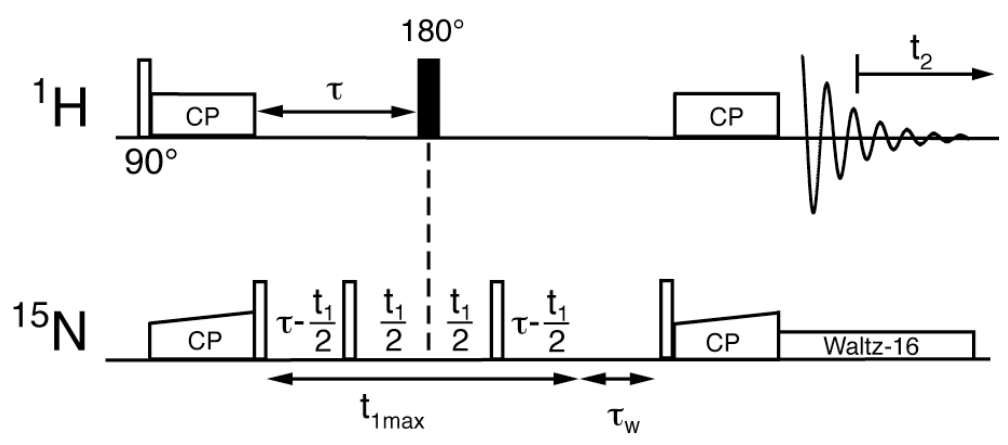

B)

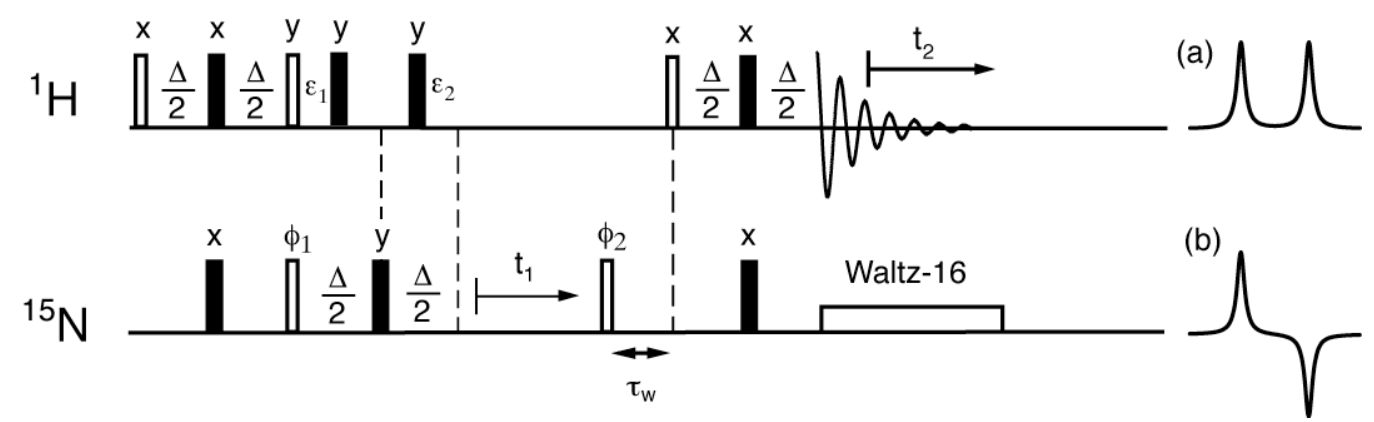

Figure 4. A) ${ }^{1} \mathrm{H},{ }^{15} \mathrm{~N}$ correlation pulse sequence. Effective suppression of the dominant water resonance was achieved by modification of the constant time (CT) experiment suggested by Zilm and co-workers. ${ }^{15}$ After magnetization transfer from ${ }^{1} \mathrm{H}$ to ${ }^{15} \mathrm{~N}$, polarization is stored along the $\mathrm{Z}$-axis during a variable delay $\left(\tau-t_{1} / 2\right)$, which precedes and follows the ${ }^{15} \mathrm{~N}$ evolution period $t_{1}$. Two delays $\left(\tau-t_{1} / 2\right)$ are required to achieve $J$ decoupling in the indirect dimension and to keep the experiment constant time with respect to water magnetization. The fixed delay $\tau_{\mathrm{w}}(60-120 \mathrm{~ms})$ which is following the CT period, is optimized for water signal supression. After back-transfer of magnetization to ${ }^{1} \mathrm{H}$, magnetization is acquired using WALTZ-16 $\left(\omega_{1}=1.6 \mathrm{kHz}\right)$ for heteronuclear scalar decoupling. ${ }^{15}$ Typical RF field strengths for $\mathrm{CP}$ are $30 \mathrm{kHz}$ and $30 \mathrm{kHz}+$ wr for the ${ }^{1} \mathrm{H}$ and ${ }^{15} \mathrm{~N}$ channel, respectively. The $\mathrm{CP}$ contact time was adjusted to $800 \mu \mathrm{sec}$. No long-range transfers were observed under these conditions. B) IPAP (in-phase anti-phase) pulse sequence for ${ }^{15} \mathrm{NH}^{\alpha} / \mathrm{H}^{\beta}$ spin-state-selection. The inphase spectrum is obtained adjusting the delays $\varepsilon_{1}=\Delta / 2$ and $\varepsilon_{2}=0$, using the phase cycle $\phi_{1}=\mathrm{x},-\mathrm{x} ; \phi_{2}=2(\mathrm{x}), 2(-$ $\mathrm{x}) ; \phi_{\mathrm{rec}}=(\mathrm{x},-\mathrm{x},-\mathrm{x}, \mathrm{x})$. The spectrum of the anti-phase spin state was recorded setting the delays according to $\varepsilon_{1}=$ $\Delta / 4$ and $\varepsilon_{2}=\Delta / 4$, while the phase cycle $\phi_{1}=\mathrm{x},-\mathrm{x} ; \phi_{2}=2(-\mathrm{y}), 2(\mathrm{y}) ; \phi_{\mathrm{rec}}=(-\mathrm{x}, \mathrm{x}, \mathrm{x},-\mathrm{x})$ was employed. The resulting spectra are schematically given next to the pulse sequence denoted as (a) and (b) for in- and anti- phase spin state, respectively. Their sum and differences yield the downfield and upfield component of the ${ }^{15} \mathrm{~N}$ doublet, respectively.

\section{REFERENCES}

1. Haeberlen, U., High Resolution NMR of Solids. Academic Press: San Diego, 1976; Vol. S1, p 1-233. 
2. Wylie, B. J.; Franks, W. T.; Rienstra, C. M., J. Phys. Chem. B 2006, 110, (22), 1092610936.

3. Hiyama, Y.; Niu, C.-H.; Silverton, J. V.; Bavoso, A.; Torchia, D. A., J. Am. Chem. Soc. 1988, 110, 2378-2383.

4. Hartzell, C. J.; Whitfield, M.; Oas, T. G.; Drobny, G. P., J. Am. Chem. Soc. 1987, 109, (20), 5966-5969.

5. Chekmenev, E. Y.; Zhang, Q.; Waddell, K. W.; Mashuta, M. S.; Wittebort, R. J., J. Am. Chem. Soc. 2004, 126, 379-384.

6. Bielecki, A.; Burum, D. P., J. Magn. Res. 1995, 116, (2), 215-220.

7. Dvinskikh, S. V.; Castro, V.; Sandstrom, D., Magn. Res. Chem. 2004, 42, (10), 875881.

8. Stringer, J. A.; Bronnimann, C. E.; Mullen, C. G.; Zhou, D. H. H.; Stellfox, S. A.; Li, Y.; Williams, E. H.; Rienstra, C. M., J. Magn. Res. 2005, 173, (1), 40-48.

9. de Lacaillerie, J. B. D.; Jarry, B.; Pascui, O.; Reichert, D., Solid State Nucl. Magn. Reson. 2005, 28, (2-4), 225-232.

10. Li, C. G.; Mo, Y. M.; Hu, J.; Chekmenev, E.; Tian, C. L.; Gao, F. P.; Fu, R. Q.; Gor'kov, P.; Brey, W.; Cross, T. A., J. Magn. Reson. 2006, 180, (1), 51-57.

11. Hartel, A. J.; Lankhorst, P.; Altona, C., Eur. J. Biochem. 1982, 129, 343-357.

12. Orbons, L. P. M.; van der Marel, G. A.; van Boom, J. H.; Altona, C., Eur. J. Biochem. 1987, 170, 225-239.

13. Ottiger, M.; Delaglio, F.; Bax, A., J. Magn. Reson. 1998, 131, 373-378.

14. Andersson, P.; Weigelt, J.; Otting, G., J. Biomol. NMR 1998, 12, (3), 435-441.

15. Paulson, E. K.; Morcombe, C. R.; Gaponenko, V.; Dancheck, B.; Byrd, R. A.; Zilm, K. W., J. Am. Chem. Soc. 2003, 125, 15831-15836. 